\title{
Spectroscopic mode identification of $\gamma$ Doradus stars
}

\author{
E. Brunsden ${ }^{1,2, \star}$, K. R. Pollard ${ }^{2}$, P. L. Cottrell ${ }^{2}$, D. J. Wright ${ }^{3}$, and P. De Cat ${ }^{4}$ \\ ${ }^{1}$ Department of Physics, University of York, York, UK \\ ${ }^{2}$ Department of Physics and Astronomy, University of Canterbury, Christchurch, New Zealand \\ ${ }^{3}$ Department of Astrophysics, University of New South Wales, Sydney, Australia \\ ${ }^{4}$ Royal Observatory of Belgium, Brussels, Belgium
}

\begin{abstract}
The g-mode pulsations in $\gamma$ Doradus stars are identified using time-series colour photometry and high-resolution spectroscopy. For 22 class members the pulsational frequencies and modes are compared. Ground-based spectroscopic and photometric results show good agreement. The prevalence of $(1,|1|)$ modes is noted and examined.
\end{abstract}

\section{Introduction}

The $\gamma$ Doradus class of stars is of particular interest in asteroseismology. They are defined as A-F type main sequence (or close to main sequence) stars that display pulsational gravity-modes (g-modes) of high radial order. These g-modes probe the deep interior of the stars and thus provide us with a tool to determine the structure and evolutionary changes that occur in regions as deep as the core.

For a successful asteroseismic study, two conditions have to be satisfied: a sufficient number of pulsation modes should be observed and the modes should be well identified. This information feeds into and refines theoretical stellar models as well as revealing new insights into the class. We present a summary of mode identifications found in ground-based spectroscopic and photometric studies of class members.

\section{Spectroscopic methods}

The MUSICIAN program uses observations that were primarily taken from the 1-metre McLellan telescope at University of Canterbury Mt John Observatory (UCMJO), New Zealand. Spectra were obtained on the High Efficiency and Resolution Canterbury University Large Echelle Spectrograph. Multi-site data were also used where possible. Frequencies were found by a Fourier analysis of the moments of the line profile. A two-dimensional analysis of the line profile was done using the pixelby-pixel method. Mode identification was done by the Fourier Parameter Fit method in the software package FAMIAS ([1]) which models frequencies, amplitudes and phases of the representative line profile to determine a mode for each frequency. Performing the fit with several frequencies and modes simultaneously gives some basic stellar parameters.

^emily.brunsden@gmail.com 


\section{Summary of results}

A total of 22 stars have been analysed for modes ${ }^{1}$, of which two are binary stars and one is a hybrid $\delta$ Scuti $/ \gamma$ Doradus star. Nine stars have photometric identifications of $l$ and 16 have full $(l, m)$ identifications. Of the 22 stars, 12 have had full mode identifications as part of the MUSICIAN programme using spectra from UCMJO. Despite the small numbers of classifications, there are enough now known to identify some global features and compare these to other identifications.

In colour photometry 33 modes have been identified, split almost equally between $l=1$ modes (16) and $l=2$ modes (17). The spectroscopic identifications (48) have $16(1,|1|)$ modes and $9 l=2$ modes, which are almost exclusively $(2,-2)$. There is no apparent correlation between the presence of retrograde modes and $v \sin i$ of a star. Stars with low to mid observed rotation speeds $\left(15 \mathrm{~km} \mathrm{~s}^{-1}<\right.$ $v \sin i<80 \mathrm{~km} \mathrm{~s}^{-1}$ ) are preferred for spectroscopic mode identification due to their broadened line profiles but minimum rotational distortion.

Only three stars have both photometric and spectroscopic mode identifications and for two stars these agree for a total of six frequencies, suggesting good agreement between the methods. There is not presently good agreement between ground-based and space-based observations (e.g., [2]), although only a few stars have such observations.

Observations to date suggest a prevalence of $(1,|1|)$ modes in $\gamma$ Doradus stars ([3-5]). This appears to also extend to colour photometry methods. The $15(1,1)$ modes identified here appear to be clustered with an average of around $1.4 \mathrm{~d}^{-1}$. Retrograde $(1,-1)$ modes are split between higher and lower frequencies. Other modes identified have a higher average of approximately $2.5 \mathrm{~d}^{-1}$ and retrograde modes are all much lower than this. A $(1,1)$ mode may arise from observational bias due to larger surface deformation. It is also possible the frequency is linked to the proximity of the rotational frequency, constraining the light maxima to once per rotation cycle. This is supported by the frequency distinction. In addition, stars with high inclinations will have higher observed amplitudes for sectoral modes and those with low inclinations, higher-amplitude tesseral and zonal modes ([6]). For rotating stars, the presence of an equatorial wave-guide ([7]) would suppress the tesseral modes and thus only the stars with sectoral modes would be classified as $\gamma$ Doradus stars.

The analysis of these stars is ongoing as we look for observational patterns in the modes from both ground-based and space-based observations to improve our understanding of these complex stars.

Acknowledgments: This work was supported by the Marsden Fund through the Royal Society of New Zealand. Mode identification results obtained with the software package FAMIAS developed in the framework of the FP6 European Coordination action HELAS (http://www.helas-eu.org/).

\section{References}

[1] W. Zima, Communications in Asteroseismology 157, 387 (2008)

[2] E. Brunsden, K.R. Pollard, P.L. Cottrell, K. Uytterhoeven, D.J. Wright, P. De Cat, MNRAS 447, 2970 (2015)

[3] L.A. Balona, J.A. Guzik, K. Uytterhoeven, J.C. Smith, P. Tenenbaum, J.D. Twicken, MNRAS 415, 3531 (2011)

[4] E. Brunsden, K.R. Pollard, P.L. Cottrell, D.J. Wright, P. De Cat, MNRAS 427, 2512 (2012)

[5] T. Van Reeth, A. Tkachenko, C. Aerts, A\&A 593, A120 (2016)

[6] D.R. Reese, V. Prat, C. Barban, C. Van't Veer-Menneret, K.B. MacGregor, A\&A 550, A77 (2013)

[7] R.H.D. Townsend, MNRAS 343, 125 (2003)

\footnotetext{
${ }^{1} \mathrm{~A}$ full table of pulsational frequencies and mode identifications is available on request or can be found in Brunsden et al. (in preparation).
} 